\title{
Clinical Relevance of Pleural Effusion in Patients with Pulmonary Embolism
}

\author{
Sun Ha Choi ${ }^{a}$ Seung-Ick Cha ${ }^{a}$ Kyung-Min Shin ${ }^{b}$ Jae-Kwang Lim ${ }^{b}$

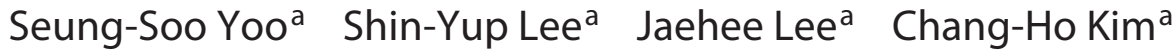 \\ Jae-Yong Park ${ }^{a}$ Deok Heon Lee ${ }^{c}$
}

Departments of ${ }^{\mathrm{a}}$ Internal Medicine, ${ }^{\mathrm{b}}$ Radiology and ${ }^{\mathrm{c}}$ Thoracic and Cardiovascular Surgery, School of Medicine, Kyungpook National University, Daegu, South Korea

\section{Keywords \\ Computed tomography · Pleural effusion · Pulmonary embolism · Pulmonary infarction}

\begin{abstract}
Background: Data regarding pleural effusion due to pulmonary embolism (PE) are limited. Objectives: The aim of this study was to investigate the clinical characteristics of PE patients with pleural effusion caused by PE. Methods: Patients with PE were retrospectively analyzed and divided into 2 groups based on computed tomography: a group with pleural effusion due to PE (effusion group) and a group without pleural effusion (control group). Clinical characteristics were compared between the 2 groups. Results: The study population consisted of the effusion group $(n=127)$ and the control group $(n=651)$. Serum C-reactive protein (CRP) level was significantly higher in the effusion group than in the control group. The percentages of high-risk Simplified PE Severity Index (57 vs. 47\%, $p=0.008$ ), central PE (84 vs. $73 \%, p=0.013$ ), right ventricular dilation (45 vs. $36 \%$, $p=0.053$ ), and pulmonary infarction (40 vs. $8 \%, p<0.001$ ) were higher in the effusion group than in the control group. Multivariate analysis demonstrated that pulmonary infarction (odds ratio [OR] 6.20, 95\% confidence interval [CI] 3.49-
\end{abstract}

10.91, $p<0.001$ ) and CRP level (OR 1.05, 95\% Cl 1.101-1.09, $p=0.023$ ) were independent predictors of pleural effusion due to PE. The presence of pleural effusion was not a predictor of short-term outcomes or length of hospital stay. Conclusions: Patients with more severe PE are likely to have pleural effusion caused by PE. However, pleural effusion was not a proven predictor of short-term outcome or length of hospital stay. Pulmonary infarction and CRP levels were independent risk factors for the development of pleural effusion.

c) 2017 S. Karger AG, Basel

\section{Introduction}

Pulmonary embolism (PE) is the fourth leading cause of pleural effusion in the United States, after congestive heart failure, parapneumonic effusion, and malignancy [1], and it is also considered one of the leading causes among patients with undiagnosed pleural effusion [2]. Among patients with PE, the percentage of those with pleural effusion varies with the assessment method and study population: $23-52 \%$ of those assessed by chest radiography have pleural effusion [3-6], as do $20-55 \%$ of those assessed by computed tomography (CT) $[4,7,8]$.

\section{KARGER}

(C) 2017 S. Karger AG, Basel

E-Mail karger@karger.com

www.karger.com/res
Seung-Ick Cha, MD

Department of Internal Medicine

Kyungpook National University Hospital

130 Dongdeok-ro, Jung-gu, Daegu 41944 (South Korea)

E-Mail sicha@knu.ac.kr 
As the amount of pleural effusion caused by PE is usually small, diagnostic thoracentesis is not routinely performed [9]. Several studies have been dedicated to analyze pleural fluid in PE patients with pleural effusion $[4,10,11]$. Many patients had bloody or blood-tinged pleural effusions [4, 9-11], and most pleural effusions due to $\mathrm{PE}$ are exudates $[4,9,10]$. A few studies have evaluated the clinical characteristics of PE patients with pleural effusion [5, 12]. However, data regarding clinical comparisons between PE patients who have pleural effusion and PE patients who do not have pleural effusion are limited [7]. In addition, there is no recent study of patients with pleural effusion caused by PE but no other comorbid condition [6].

The aim of this study was to investigate and compare the clinical characteristics of PE patients with pleural effusion caused by PE and with PE patients without pleural effusion, and to determine predictors of pleural effusion due to PE. Finally, we tried to elucidate the clinical relevance of pleural effusion in PE patients.

\section{Methods}

\section{Study Design}

This study was retrospectively conducted in a tertiary referral center, Kyungpook National University Hospital (KNUH) in Daegu, South Korea. Patients with PE who had been diagnosed by using multidetector-row CT between March 2003 and December 2015 were enrolled. The presence and causes of pleural effusion were determined by the consensus of 2 chest physicians (S.H.C. and S.-I.C.) on the basis of CT scans and medical records. Pleural effusion ipsilateral to pulmonary emboli or adjacent to pulmonary infarcts that could not be explained by any other condition was defined as pleural effusion due to PE. Patients with other conditions that were likely to have caused pleural effusion were excluded. Clinical characteristics, laboratory findings, and CT-based parameters were compared between patients with pleural effusion due to PE and PE patients without pleural effusion, and potential predictors of pleural effusion caused by PE were examined. This study was approved by the Institutional Review Board of the $\mathrm{KNUH}$, which waived the requirement for written informed consent because of the retrospective nature of the study.

\section{Data Collection}

Demographic data, symptoms, vital signs, and predisposing or comorbid conditions were examined. PE was defined as unprovoked if no reversible provoking risk factors had occurred; these factors included surgery, trauma, active cancer, pregnancy and puerperium within 3 months of the event, and immobilization (bed rest for most of the day for $\geq 3$ consecutive days) within 1 month of the event [13]. Ratings based on the Simplified PE Severity Index (sPESI) [14] were retrospectively calculated for patients before 2011 and have been prospectively recorded in their medical records since 2011. Outcome parameters, including in-hospital mortality (PE-related or PE-unrelated), adverse outcomes, and recurrence of venous thromboembolism were reviewed. As in a previ- ous study, an adverse outcome was defined as a PE-related in-hospital death or PE resulting in a serious clinical condition requiring intensive care treatment, such as inotropic support and mechanical ventilation, impending respiratory failure or refractory hypoxia, cardiopulmonary resuscitation, or secondary thrombolysis [15]. A PE-related in-hospital death was defined as an inhospital death that met one of the following criteria: (1) objective evidence of death directly caused by PE, and (2) death that could not be attributed to other causes and in which PE could not be excluded [15].

Blood samples were obtained within $12 \mathrm{~h}$ of the initial visit. Blood laboratory data, including erythrocyte sedimentation rate (ESR) and levels of N-terminal-pro-B-type natriuretic peptide (NT-proBNP), troponin I, C-reactive protein (CRP), and procalcitonin, were reviewed. Arterial blood gas analysis data consisted of the partial pressure of oxygen in arterial blood $\left(\mathrm{PaO}_{2}\right)$, the partial pressure of carbon dioxide in arterial blood $\left(\mathrm{PaCO}_{2}\right)$, the inspired oxygen fraction $\left(\mathrm{FiO}_{2}\right)$, and the $\mathrm{PaO}_{2} / \mathrm{FiO}_{2}$ ratio.

\section{Radiological Evaluation}

As in an earlier study [16], in this study, a PE was diagnosed on CT images as a sharply delineated filling defect that was seen in a pulmonary artery in at least 2 consecutive image sections and that either was located centrally within the vessel or formed acute angles with the arterial wall. PE-involved largest pulmonary arteries were determined as follows: pulmonary trunk, pulmonary (right or left pulmonary artery), interlobar or lobar, segmental, and subsegmental. Central PE was diagnosed when thrombi were visualized in the lobar or more proximal pulmonary arteries. The diameters of the right ventricle (RV) and left ventricle (LV) were measured at their widest points between the inner surface of the free wall and the surface of the interventricular septum; the RV diameter was typically measured at the level of the tricuspid valve, and the LV diameter was measured at the level of the mitral valve [17]. Subsequently, the RV/LV diameter ratios were calculated. An RV was considered dilated if the RV/LV diameter ratio measured on a CT scan equaled or exceeded 1 [15]. The extent of pleural effusion was assessed as follows: small, $<3 \mathrm{~cm}$ at maximal depth; moderate, $3-5 \mathrm{~cm}$; and large, $>5 \mathrm{~cm}$ [18]. On the basis of modified criteria of a prior study $[19,20]$, a pulmonary infarction was defined as the presence of a peripheral consolidation in the region of a segmental or subsegmental PE.

\section{Statistical Analysis}

Statistical analysis was performed by using IBM SPSS Statistics for Windows, version 23.0 (IBM Corp., Armonk, NY, USA). Data were expressed as means \pm standard deviations or medians with interquartile ranges for continuous variables and as numbers with percentages for categorical variables. Between groups, continuous variables were compared by using the Student $t$ test and MannWhitney $U$ test for nonnormally distributed data. Categorical variables were compared by using the $\chi^{2}$ test. Multiple logistic regression analysis was used to identify predictors of pleural effusion. The Hosmer-Lemeshow test was used as a goodness-of-fit test to assess the fit of logistic regression models. Length of hospital stay (LOS) was analyzed by using the Kaplan-Meier method; patients who died were censored from this analysis. Independent prognostic factors for LOS were identified by using a Cox regression model. $p$ values $<0.05$ were considered statistically significant. 
Fig. 1. Patient inclusion flowchart. Of 881 patients with pulmonary embolism on computed tomography, 103 patients with other causes than pleural effusion were excluded.

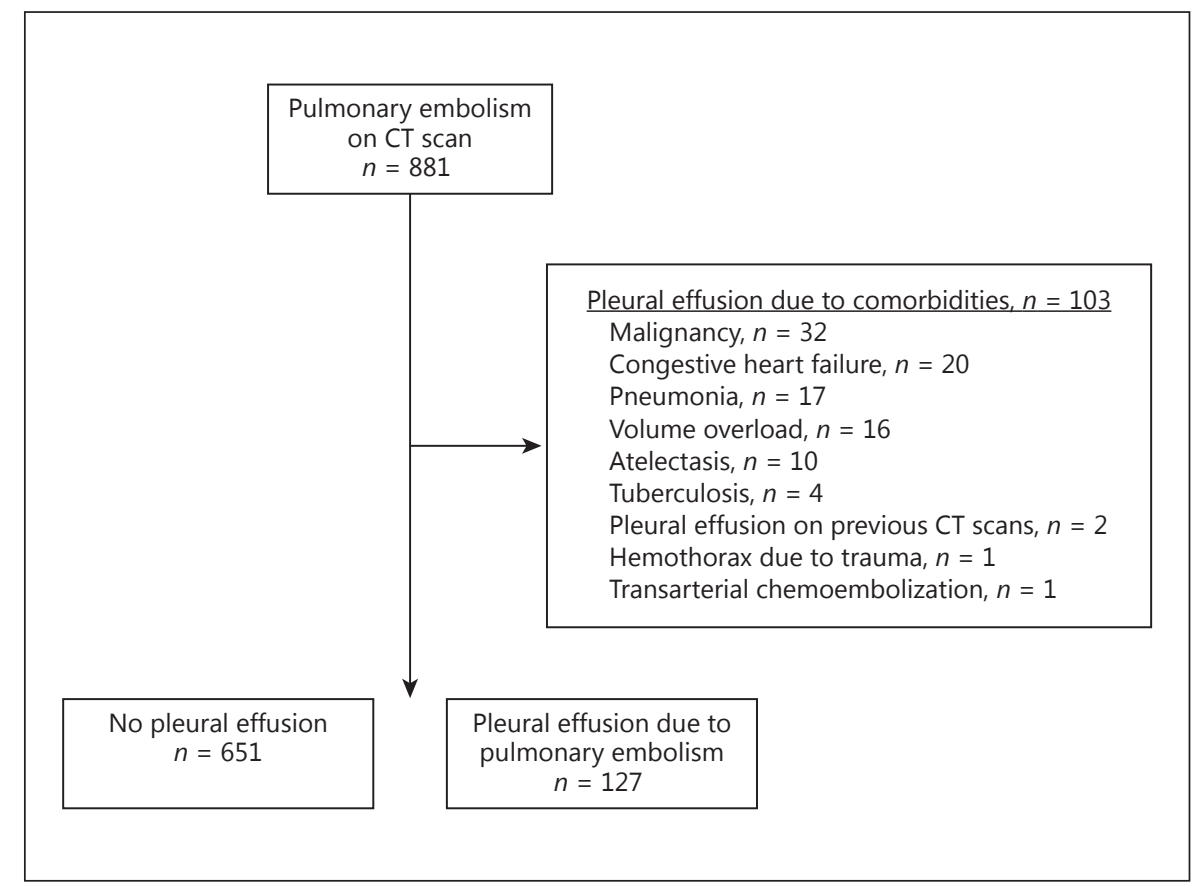

\section{Results}

\section{Clinical Characteristics}

On the basis of CT scans, a total of 881 patients were identified as having PE. Pleural effusion was identified in 230 patients (26\%), and 103 patients had pleural effusion caused by conditions other than PE: malignancies (32 [14\%]), congestive heart failure (20 [9\%]), and pneumonia (17 [7\%]) (Fig. 1). After excluding the patients with causes of pleural effusion other than PE, the remaining patients were divided into the effusion group $(n=127$; patients who had pleural effusion due to $\mathrm{PE}$ ) and the control group ( $n=651$; patients who did not have pleural effusion). The extent of pleural effusion was small in most patients (112 [88\%]), moderate in $13(10 \%)$, and large in 2 (2\%). Except for body mass index values, demographic data did not significantly differ between the 2 groups (Table 1). Pleuritic chest pain (31 [24\%] vs. 29 [5\%], $p<$ $0.001)$ and hemoptysis $(14[11 \%]$ vs. $16[3 \%], p<0.001)$ were significantly more common in the effusion group than in the control group, whereas unsuspected PE was significantly less common in the effusion group than in the control group (26 [21\%] vs. $201[31 \%], p=0.017)$. No significant differences in risk factors for venous thromboembolism existed between the 2 groups. The frequencies of hypotension (7 [6\%] vs. 13 [2\%], $p=0.022$ ) and tachycardia $(24[19 \%]$ vs. $67[10 \%], p=0.004)$ were significant- ly higher in the effusion group than in the control group. According to the sPESI, high-risk patients comprised a significantly greater percentage of the effusion group than of the control group (72 [57\%] vs. 305 [47\%], $p=$ 0.008 ). The patients of the effusion group significantly more often underwent systemic thrombolysis than those of the control group (14 [11.0\%] vs. 36 [5.5\%], $p=0.028)$, whereas most patients of both groups received anticoagulation. PE-related in-hospital deaths (4 [3\%] vs. 5 [1\%], $p<0.001)$ were significantly more common and adverse outcomes (18 [14\%] vs. 59 [9\%], $p=0.078)$ tended to be more common in the effusion group than in the control group.

\section{Laboratory and Computed Tomographic Findings}

Laboratory findings are presented in Table 2. Levels of CRP $(6.28 \mathrm{mg} / \mathrm{dL}[1.56-11.62 \mathrm{mg} / \mathrm{dL}]$ vs. $1.26 \mathrm{mg} / \mathrm{dL}$ $[0.44-4.43 \mathrm{mg} / \mathrm{dL}], p<0.001)$ and ESR $(26 \mathrm{~mm} / \mathrm{h}[9-50$ $\mathrm{mm} / \mathrm{h}$ ] vs. $18 \mathrm{~mm} / \mathrm{h}[8-86 \mathrm{~mm} / \mathrm{h}], p=0.026)$ were significantly higher in the effusion group than in the control group. Serum NT-proBNP levels tended to be higher in the effusion group, although the difference was statistically insignificant.

Central PE was significantly more common (106 [84\%] vs. 475 [73\%], $p=0.013)$ and RV dilation tended to be more common (57 [45\%] vs. 233 [36\%], $p=0.053$ ) in the effusion group than in the control group (Table 3 ). In the 
Table 1. Clinical characteristics of patients with pulmonary embolism $(n=778)$

\begin{tabular}{|c|c|c|c|}
\hline & $\begin{array}{l}\text { Effusion } \\
(n=127)\end{array}$ & $\begin{array}{l}\text { Controls } \\
(n=651)\end{array}$ & $p$ value \\
\hline Age, years & $69(51-76)$ & $69(60-75)$ & 0.116 \\
\hline Female gender & $72(56.7)$ & $368(56.5)$ & 0.973 \\
\hline Ever smoker & $39(31.5)$ & $239(37.4)$ & 0.294 \\
\hline Body mass index & $23.2 \pm 4.3$ & $24.0 \pm 3.8$ & 0.059 \\
\hline Duration of symptoms, days & $6.2 \pm 11.0$ & $9.2 \pm 25.5$ & 0.245 \\
\hline Unprovoked pulmonary embolism & $42(33.1)$ & $230(35.3)$ & 0.625 \\
\hline \multicolumn{4}{|l|}{ Presenting manifestation } \\
\hline Dyspnea & $56(44.1)$ & $275(42.2)$ & 0.699 \\
\hline Pleuritic chest pain & $31(24.4)$ & $29(4.5)$ & $<0.001$ \\
\hline Incidental finding on computed tomography scan & $26(20.5)$ & $202(31.0)$ & 0.017 \\
\hline Hemoptysis & $14(11.0)$ & $16(2.5)$ & $<0.001$ \\
\hline Leg pain or swelling & $8(6.3)$ & $98(15.1)$ & 0.009 \\
\hline Syncope & $3(2.4)$ & $8(1.2)$ & 0.322 \\
\hline General weakness & $2(1.6)$ & $5(0.8)$ & 0.378 \\
\hline Fever & $1(0.8)$ & $1(0.2)$ & 0.197 \\
\hline Incidental finding of hypoxia & $0(0)$ & $4(0.6)$ & 0.376 \\
\hline Hypotension & $0(0)$ & $2(0.3)$ & 0.532 \\
\hline \multicolumn{4}{|l|}{ Predisposing or comorbid condition } \\
\hline Immobilization & $59(46.5)$ & $275(42.2)$ & 0.525 \\
\hline Trauma or surgery & $35(27.6)$ & $170(26.1)$ & 0.735 \\
\hline Cancer & $24(18.9)$ & $148(22.7)$ & 0.341 \\
\hline Stroke & $14(11.0)$ & $73(11.2)$ & 0.950 \\
\hline Chronic pulmonary disease* & $10(7.9)$ & $72(11.0)$ & 0.285 \\
\hline Respiratory tract infection & $11(8.7)$ & $31(4.8)$ & 0.075 \\
\hline Pulmonary tuberculosis & $2(1.6)$ & $14(2.2)$ & 0.757 \\
\hline Congestive heart failure & $0(0)$ & $15(2.3)$ & 0.149 \\
\hline Hypotension, systolic blood pressure $<90 \mathrm{~mm} \mathrm{Hg}$ & $7(5.5)$ & $13(2.0)$ & 0.022 \\
\hline Tachycardia, heart rate $>110 / \mathrm{min}$ & $24(18.9)$ & $67(10.3)$ & 0.004 \\
\hline Simplified pulmonary embolism severity index, high risk & $72(56.7)$ & $305(46.8)$ & 0.008 \\
\hline Anticoagulation & $121(95.3)$ & $596(91.6)$ & 0.201 \\
\hline Systemic thrombolysis & $14(11.0)$ & $36(5.5)$ & 0.028 \\
\hline Length of hospital stay, days & $12(8-19)$ & $11(7-17)$ & 0.460 \\
\hline In-hospital mortality & $16(12.6)$ & $28(4.3)$ & $<0.001$ \\
\hline Pulmonary embolism-related in-hospital mortality & $4(3.1)$ & $5(0.8)$ & $<0.001$ \\
\hline Adverse outcome & $18(14.2)$ & $59(9.1)$ & 0.078 \\
\hline Venous thromboembolism recurrence & $9(7.1)$ & $40(6.1)$ & 0.861 \\
\hline
\end{tabular}

Data are presented as median (interquartile range), mean \pm standard deviation, or $n(\%) . *$ Chronic lung disease includes chronic obstructive pulmonary disease, asthma, bronchiectasis, idiopathic pulmonary fibrosis, pneumoconiosis, and tuberculosis-destroyed lung.

effusion group, most patients with central PE (98/106 [92.5\%]) had peripheral as well as central pulmonary emboli. The percentage of patients with pulmonary infarction was significantly higher in the effusion group than in the control group (51 [40\%] vs. $51[8 \%], p<0.001$ ).

\section{Predictors of Pleural Effusion in Patients with PE}

Candidate variables with $p$ values $<0.10$, including sPESI results, CRP levels, ESR levels, central PE, and pul- monary infarction, were chosen for multivariate analysis for predictors of pleural effusion caused by PE (HosmerLemeshow test, $p=0.387$ ). The results of multivariate analysis indicated that pulmonary infarction (odds ratio [OR] 6.20, 95\% confidence interval [CI] 3.49-10.91, $p<$ 0.001 ) and CRP levels (OR 1.05, 95\% CI 1.101-1.09, $p=$ 0.023 ) were independent predictors of pleural effusion due to PE (Table 4). 
Table 2. Laboratory findings

\begin{tabular}{lllllr}
\hline & $n$ & $\begin{array}{l}\text { Effusion } \\
(n=127)\end{array}$ & $n$ & $\begin{array}{l}\text { Controls } \\
(n=651)\end{array}$ & $p$ value \\
\hline NT-proBNP, pg/mL & 86 & $511(104-2,874)$ & 430 & $376(107-1,611)$ & 0.094 \\
Troponin I, ng/mL & 91 & $0.23 \pm 0.64$ & 502 & $0.17 \pm 0.54$ & 0.399 \\
$\mathrm{CRP}, \mathrm{mg} / \mathrm{dL}$ & 96 & $6.28(1.56-11.62)$ & 462 & $1.26(0.44-4.43)$ & $<0.001$ \\
ESR, mm/h & 85 & $26(9-50)$ & 423 & $18(8-36)$ & 0.026 \\
$\mathrm{Procalcitonin} \mathrm{ng} / \mathrm{mL}$ & 26 & $0.43 \pm 1.30$ & 101 & $0.44 \pm 1.48$ & 0.977 \\
$\mathrm{PaO}_{2} / \mathrm{FiO}, \mathrm{mm} \mathrm{Hg}$ & 87 & $342 \pm 93$ & 416 & $343 \pm 98$ & 0.943 \\
$\mathrm{PaCO}_{2}, \mathrm{~mm} \mathrm{Hg}$ & 88 & $30.0(24.9-34.2)$ & 408 & $29.5(26.5-34.0)$ & 0.871 \\
\hline
\end{tabular}

Data are presented as median (interquartile range) or mean \pm standard deviation. NT-proBNP, N-terminalpro-B-type natriuretic peptide; $\mathrm{CRP}, \mathrm{C}$-reactive protein; ESR, erythrocyte sedimentation rate; $\mathrm{PaO}_{2}$, pressure of oxygen in arterial blood; $\mathrm{PaCO}_{2}$, partial pressure of carbon dioxide in arterial blood; $\mathrm{FiO}_{2}$, inspired oxygen fraction.

Table 3. Radiologic findings

\begin{tabular}{lccr}
\hline & $\begin{array}{l}\text { Effusion } \\
(n=127)\end{array}$ & $\begin{array}{l}\text { Controls } \\
(n=651)\end{array}$ & $p$ value \\
\hline $\begin{array}{l}\text { Location of the largest pulmonary emboli } \\
\quad \text { Main or more proximal }\end{array}$ & $57(44.9)$ & $224(34.4)$ & 0.025 \\
$\quad$ Interlobar or lobar & $49(38.6)$ & $251(38.6)$ & 0.996 \\
$\quad$ Segmental & $16(12.6)$ & $137(21.0)$ & 0.028 \\
$\quad$ Subsegmental & $4(3.1)$ & $39(6.0)$ & 0.200 \\
$\quad$ Central pulmonary embolism* & $81 / 115(70.4)$ & $375(73.0)$ & 0.013 \\
Deep vein thrombosis & $51(47.5)$ & $240(479(69.2)$ & 0.187 \\
$\quad$ Proximal deep vein thrombosis & $57(44.9)$ & $233(35.8)$ & 0.483 \\
Right ventricle dilation on computed tomography & $51(40.2)$ & $51(7.8)$ & 0.053 \\
Pulmonary infarction & & $<0.001$ \\
\hline
\end{tabular}

Data are presented as $n(\%) .{ }^{*}$ Central pulmonary arteries mean lobar pulmonary artery or more proximal location.

\section{Pleural Fluid Analysis}

The results of the pleural fluid analysis are presented as online supplementary material (online suppl. Table S1; see www.karger.com/doi/10.1159/000457132 for all online suppl. material). Diagnostic thoracentesis was performed in only $13 \mathrm{PE}$ patients (10\%), and of these patients, 12 (92\%) had exudative pleural effusion. Serosanguinous fluid was noted in 9 patients (69\%), and 4 patients (31\%) had pleural fluid hematocrits of $>1 \%$. Only 2 patients underwent percutaneous catheter drainage.

\section{Predictors of Mortality, Adverse Outcomes, and LOS}

The presence of pleural effusion in PE patients was not a predictor of PE-related in-hospital mortality in multi-
Table 4. Multivariate analysis for predictors of pleural effusion due to pulmonary embolism

\begin{tabular}{lclr}
\hline & OR & $95 \%$ CI & $p$ value \\
\hline Pulmonary infarction & 6.20 & $3.49-10.91$ & $<0.001$ \\
CRP & 1.05 & $1.01-1.09$ & 0.023 \\
Central PE & 1.65 & $0.82-3.33$ & 0.159 \\
sPESI & 1.22 & $0.74-2.04$ & 0.438 \\
ESR & 1.00 & $0.97-1.01$ & 0.735
\end{tabular}

Hosmer-Lemeshow test, $p=0.387$. OR, odds ratio; CI, confidence interval; CRP, C-reactive protein; PE, pulmonary embolism; sPESI, Simplified Pulmonary Embolism Severity Index; ESR, erythrocyte sedimentation rate. 
Table 5. Predictors of pulmonary embolism-related in-hospital mortality

\begin{tabular}{|c|c|c|c|c|c|c|}
\hline & \multicolumn{3}{|c|}{ Univariate analysis } & \multicolumn{3}{|c|}{ Multivariate analysis* } \\
\hline & OR & $95 \% \mathrm{CI}$ & $p$ value & OR & $95 \% \mathrm{CI}$ & $p$ value \\
\hline Age & 1.00 & $0.96-1.05$ & 0.876 & & & \\
\hline Female gender & 0.61 & $0.16-2.29$ & 0.465 & 0.75 & $0.16-3.49$ & 0.716 \\
\hline Hypotension & 4.90 & $0.58-41.11$ & 0.144 & & & \\
\hline Tachycardia & 1.43 & $0.29-6.97$ & 0.657 & & & \\
\hline sPESI, high risk & 2.15 & $0.53-8.62$ & 0.282 & 0.85 & $0.18-4.02$ & 0.837 \\
\hline NT-proBNP & 1.00 & $1.00-1.00$ & 0.158 & 1.00 & $1.00-1.00$ & 0.521 \\
\hline Troponin I & 1.02 & $0.29-3.64$ & 0.977 & & & \\
\hline $\mathrm{PaO}_{2} / \mathrm{FiO}_{2}$ & 1.00 & $0.99-1.00$ & 0.129 & & & \\
\hline RV dilation on CT & 6.01 & $1.24-29.13$ & 0.011 & 7.15 & $0.82-62.67$ & 0.076 \\
\hline Central PE & 1.19 & $0.24-5.74$ & 0.835 & & & \\
\hline Pleural effusion & 4.20 & $1.11-15.87$ & 0.034 & 3.35 & $0.70-15.95$ & 0.129 \\
\hline
\end{tabular}

OR, odds ratio; CI, confidence interval; sPESI, Simplified Pulmonary Embolism Severity Index; NT-proBNP, $\mathrm{N}$-terminal-pro-B-type natriuretic peptide; $\mathrm{PaO}_{2}$, pressure of oxygen in arterial blood; $\mathrm{FiO}_{2}$, inspired oxygen fraction; RV, right ventricle; CT, computed tomography; PE pulmonary embolism. * Hosmer-Lemeshow test, $p=0.871$.

Table 6. Multivariate analysis for predictors of adverse outcome due to pulmonary embolism

\begin{tabular}{|c|c|c|c|c|c|c|}
\hline & \multicolumn{3}{|c|}{ Univariate analysis } & \multicolumn{3}{|c|}{ Multivariate analysis* } \\
\hline & $\overline{\mathrm{OR}}$ & $95 \% \mathrm{CI}$ & $p$ value & OR & $95 \% \mathrm{CI}$ & $p$ value \\
\hline Age & 1.02 & $1.00-1.04$ & 0.079 & & & \\
\hline Female gender & 2.05 & $1.22-3.44$ & 0.006 & 1.77 & $0.91-3.43$ & 0.092 \\
\hline Hypotension & 19.96 & $7.69-51.82$ & $<0.001$ & & & \\
\hline Tachycardia & 2.04 & $1.19-3.50$ & 0.009 & & & \\
\hline sPESI, high risk & 2.94 & $1.76-4.93$ & $<0.001$ & 2.01 & $1.08-3.77$ & 0.029 \\
\hline NT-proBNP & 1.00 & $1.00-1.00$ & $<0.001$ & 1.00 & $1.00-1.00$ & 0.005 \\
\hline Troponin I & 1.33 & $0.96-1.84$ & 0.084 & 1.16 & $0.81-1.66$ & 0.424 \\
\hline $\mathrm{PaO}_{2} / \mathrm{FiO}_{2}$ & 0.99 & $0.99-0.99$ & $<0.001$ & & & \\
\hline RV dilation on CT & 3.34 & $2.05-5.45$ & $<0.001$ & 2.44 & $1.27-4.71$ & 0.008 \\
\hline Central PE & 3.70 & $1.67-8.19$ & $<0.001$ & 1.38 & $0.51-3.78$ & 0.527 \\
\hline Pleural effusion & 1.66 & $0.94-2.92$ & 0.078 & 1.44 & $0.71-2.95$ & 0.314 \\
\hline
\end{tabular}

OR, odds ratio; CI, confidence interval; sPESI, Simplified Pulmonary Embolism Severity Index; NT-proBNP, $\mathrm{N}$-terminal-pro-B-type natriuretic peptide; $\mathrm{PaO}_{2}$, pressure of oxygen in arterial blood; $\mathrm{FiO}_{2}$, inspired oxygen fraction; RV, right ventricle; CT, computed tomography; PE, pulmonary embolism. * Hosmer-Lemeshow test, $p=0.308$.

variate analysis, despite statistical significance in univariate analysis (Table 1, 5). In addition, pleural effusion was not a predictor of adverse outcomes of PE (Table 6) or LOS (online suppl. Table S2).

\section{Discussion}

The main findings from the present study were summarized as follows. Compared to the control group, the effusion group had higher rates of hypotension, tachycardia, high-risk sPESI, central PE, and RV dilation. The 
presence of pleural effusion due to $\mathrm{PE}$ was not a predictor of PE-related in-hospital mortality, adverse outcomes, and LOS in PE patients. In a multivariate analysis, only 2 independent risk factors for pleural effusion due to $\mathrm{PE}$ were found: pulmonary infarction and serum CRP levels.

Of the clinical characteristics, hypotension, tachycardia, and high-risk sPESI occurred more commonly among PE patients with pleural effusion. In univariate analysis, PE-related in-hospital mortality was significantly more frequent and adverse outcomes tended to be more frequent in the effusion group, although the differences did not reach statistical insignificance in multivariate analysis. These findings suggest that pleural effusion caused by PE is associated with more severe PE. In addition, NT-proBNP levels also tended to be higher in the effusion group. Moreover, central PE and RV dilation were found more often in the effusion group. Nonetheless, a previous study reported that the location of the clots and the number of arteries involved were not predictors of pleural effusion [12]. However, that study was conducted on PE patients with all-cause pleural effusion, not on patients with pleural effusion caused by PE. Despite its association with more severe PE, the development of pleural effusion was not an independent predictor of short-term outcome of PE, such as PE-related in-hospital mortality or adverse outcomes, in the present study.

As in a previous report [7], in the present study, pulmonary infarction was more commonly found in PE patients with pleural effusion than in PE patients without pleural effusion. Hemoptysis and pleuritic chest pain suggestive of pulmonary infarction were more frequent in the effusion group. In a previous study, the pulmonary infarction group had a higher prevalence of pleural effusion than did the isolated dyspnea group and the circulatory collapse group [21]. In another study [4], however, wedge-shaped opacities that suggest pulmonary infarction occurred in equal proportions of patients with pleural effusion and those without the condition. However, that study included all PE patients with pleural effusion regardless of the cause of effusions. Hemorrhagic necrosis of an infarct induces or worsens pleural effusion by aggravating inflammatory reactions and causing hemorrhage in the pleural space [11], a finding that is supported by the higher CRP and ESR levels seen in the effusion group in our study. Therefore, pulmonary infarction contributes to the formation of pleural effusion in PE patients, although it is not necessary for the development of pleural effusion. However, as some PE patients had infectious or inflammatory diseases, the possibility that these comorbid conditions might influence those findings could not be excluded.

Pleural Effusion in Pulmonary Embolism
The extent of pleural effusion caused by PE was small in most cases, as was seen in previous studies $[9,22,23]$. Two patients with large-extent pleural effusion underwent pleural drainage. Of the 13 patients who underwent diagnostic thoracentesis, 12 (92\%) had exudative pleural effusion. This finding matched the classical concept that pleural effusion in PE is exudate $[4,9,10]$. The underlying mechanisms of pleural effusion in PE can be explained as follows. First, cytokines such as vascular endothelial growth factor or inflammatory mediators released from platelet-rich emboli increase the permeability of the pulmonary capillaries. The increased interstitial fluid of the lung passes through the visceral pleura and leads to an increase in pleural fluid [23]. Second, ischemia of pulmonary capillaries distal to emboli may also increase permeability, although this occurrence may play a minor role [9]. These mechanisms may also explain why pulmonary infarction and CRP levels were independent predictors of pleural effusion due to PE. Of the 13 patients who underwent thoracentesis, 1 had transudative pleural effusion. The formation of transudates can be explained by an increase in the systemic venous pressure at the parietal pleural surface secondary to pulmonary hypertension and increase in the right ventricular pressure $[9,22]$. Nevertheless, transudate in patients with $\mathrm{PE}$ is more likely to be due to other causes, such as congestive heart failure rather than pulmonary hypertension due to PE [9].

Given that pulmonary infarction was more frequently observed in the effusion, small peripheral pulmonary emboli are more likely to cause pleural effusion than central PE [20]. However, in the present study, central PE was more common in the effusion group than in the control group. This discrepancy can be explained by the finding that most patients with central PE had peripheral as well as central emboli, supporting that patients with pleural effusion exhibited higher clot burden and more severe PE.

This study has several limitations. First, as this is a retrospective study, selection bias was inevitable. Although 2 chest physicians examined and determined the causes of pleural effusion by consensus, the chance of misclassification due to retrospective design should be considered. Second, as PE patients who had been diagnosed only by CT were included in the present study, the exclusion of patients diagnosed by perfusion/ventilation scan or ultrasound for deep vein thrombosis and clinical prediction score might affect the results, Third, there have been no widely accepted diagnostic criteria for pulmonary infarction, although those used in a previous study [20] were adopted in this study. Fourth, missing laboratory data, 
such as NT-proBNP levels, could have influenced our results. Lastly, the possibility that PE-specific therapy may have affected the clinical outcome could not be excluded.

In conclusion, patients with more severe PE are likely to have pleural effusion caused by PE. However, pleural effusion was not proven to predict short-term outcomes or LOS in PE patients. Further studies with a larger patient population are required to explain why effusions may be a marker of severity but not outcome of PE. Additionally, pulmonary infarction and CRP levels were independent risk factors for the development of pleural effusion due to PE.

\section{References}

1 Light RW: Clinical practice. Pleural effusion. New Engl J Med 2002;346:1971-1977.

2 Light RW: The undiagnosed pleural effusion. Clin Chest Med 2006;27:309-319.

3 Elliott CG, Goldhaber SZ, Visani L, DeRosa $\mathrm{M}$ : Chest radiographs in acute pulmonary embolism. Results from the International Cooperative Pulmonary Embolism Registry. Chest 2000;118:33-38.

4 Porcel JM, Madronero AB, Pardina M, Vives M, Esquerda A, Light RW: Analysis of pleural effusions in acute pulmonary embolism: radiological and pleural fluid data from $230 \mathrm{pa}-$ tients. Respirology 2007;12:234-239.

5 Stein PD, Terrin ML, Hales CA, Palevsky HI, Saltzman HA, Thompson BT, et al: Clinical, laboratory, roentgenographic, and electrocardiographic findings in patients with acute pulmonary embolism and no pre-existing cardiac or pulmonary disease. Chest 1991; 100:598-603.

6 Bynum LJ, Wilson JE 3rd: Radiographic features of pleural effusions in pulmonary embolism. Am Rev Respir Dis 1978;117:829-834.

7 Liu M, Cui A, Zhai ZG, Guo XJ, Li M, Teng LL, et al: Incidence of pleural effusion in patients with pulmonary embolism. Chin Med J 2015;128:1032-1036.

8 Karabulut N, Kiroglu Y: Relationship of parenchymal and pleural abnormalities with acute pulmonary embolism: CT findings in patients with and without embolism. Diagn Interv Radiol 2008;14:189-196.
9 Findik S: Pleural effusion in pulmonary embolism. Curr Opin Pulm Med 2012;18:347354.

10 Romero Candeira S, Hernandez Blasco L, Soler MJ, Munoz A, Aranda I: Biochemical and cytologic characteristics of pleural effusions secondary to pulmonary embolism. Chest 2002;121:465-469.

11 Bynum LJ, Wilson JE 3rd: Characteristics of pleural effusions associated with pulmonary embolism. Arch Intern Med 1976;136:159162.

12 Yap E, Anderson G, Donald J, Wong CA, Lee YC, Sivakumaran P: Pleural effusion in patients with pulmonary embolism. Respirology 2008; 13:832-836.

13 Choi KJ, Cha SI, Shin KM, Lee J, Hwangbo Y, Yoo SS, et al: Prevalence and predictors of pulmonary embolism in Korean patients with exacerbation of chronic obstructive pulmonary disease. Respiration 2013;85:203-209.

14 Jimenez D, Aujesky D, Moores L, Gomez V, Lobo JL, Uresandi F, et al: Simplification of the pulmonary embolism severity index for prognostication in patients with acute symptomatic pulmonary embolism. Arch Intern Med 2010;170:1383-1389.

15 Choi KJ, Cha SI, Shin KM, Lim J, Yoo SS, Lee $\mathrm{J}$, et al: Prognostic implications of computed tomographic right ventricular dilation in patients with acute pulmonary embolism. Thromb Res 2014;133:182-186.
16 Gladish GW, Choe DH, Marom EM, Sabloff BS, Broemeling LD, Munden RF: Incidental pulmonary emboli in oncology patients: prevalence, CT evaluation, and natural history. Radiology 2006;240:246-255.

17 Araoz PA, Gotway MB, Harrington JR, Harmsen WS, Mandrekar JN: Pulmonary embolism: prognostic CT findings. Radiology 2007;242:889-897.

18 Shah AA, Davis SD, Gamsu G, Intriere L: Parenchymal and pleural findings in patients with and patients without acute pulmonary embolism detected at spiral CT. Radiology 1999;211:147-153.

19 Revel MP, Triki R, Chatellier G, Couchon S, Haddad N, Hernigou A, et al: Is It possible to recognize pulmonary infarction on multisection CT images? Radiology 2007;244:875882.

20 Cha SI, Shin KM, Lee J, Hwangbo Y, Yoo SS, Lee SY, et al: Clinical relevance of pulmonary infarction in patients with pulmonary embolism. Thromb Res 2012;130:e1-e5.

21 Stein PD, Henry JW: Clinical characteristics of patients with acute pulmonary embolism stratified according to their presenting syndromes. Chest 1997;112:974-979.

22 Light RW: Pleural effusion in pulmonary embolism. Semin Respir Crit Care Med 2010;31: 716-722.

23 Porcel JM: pleural effusion due to pulmonary embolism. Curr Opin Pulm Med 2008;14: 337-342. 